See discussions, stats, and author profiles for this publication at: https://www.researchgate.net/publication/328568029

\title{
Novel Reactivation Allows Effective Reuse of Nafion Super-acid Nano-catalyst
}

Article in Applied Catalysis A: General · October 2018

DOI: 10.1016/j.apcata.2018.10.035

CITATIONS

READS

0

22

5 authors, including:

Ekaterina Ponomareva

Semenov Institute of Chemical Physics, Russian Academy of Sciences

15 PUBLICATIONS 15 CITATIONS

SEE PROFILE

6. Mariví Morales

Spanish National Research Council

13 PUBLICATIONS 46 CITATIONS

SEE PROFILE

Some of the authors of this publication are also working on these related projects:

Project Energy Harvest Research Study View project

Project Waste plastics back to the supply chain View project

Daan Wigger

1 PUBLICATION O CITATIONS

SEE PROFILE

Ignacio Melian Cabrera

Aston University

98 PUBLICATIONS 2,050 CITATIONS

SEE PROFILE 


\title{
Novel Reactivation Allows Effective Reuse of Nafion Super-acid Nano-catalyst
}

\author{
Ekaterina Ponomareva, ${ }^{1 \perp}$ Marco-Antonio López-Martínez, ${ }^{2 \perp}$ Daan Wigger, ${ }^{3 \perp}$ \\ Maria V. Morales, ${ }^{4}$ and Ignacio Melián-Cabrera ${ }^{3,4 *}$ \\ ${ }^{1}$ Semenov Institute of Chemical Physics, Department of Kinetics and Catalysis, Laboratory of \\ Heterogeneous Catalysis, Russian Academy of Sciences, 119991 Moscow, Russian Federation. \\ ${ }^{2}$ Departamento de Ciencias Básicas, División de Ciencias Básicas e Ingeniería, Universidad \\ Autónoma Metropolitana-Unidad Azcapotzalco, Av. San Pablo 180, Col. Reynosa Tamaulipas, Del. \\ Azcapotzalco 02200, Mexico City, Mexico. \\ ${ }^{3}$ Chemical Reaction Engineering (ITM-FWN), University of Groningen, Nijenborgh 4, 9747 AG \\ Groningen, The Netherlands. \\ ${ }^{4}$ Aston Institute of Materials Research, School of Engineering and Applied Science, Aston University, \\ Aston Triangle, Birmingham, B4 7ET, United Kingdom.

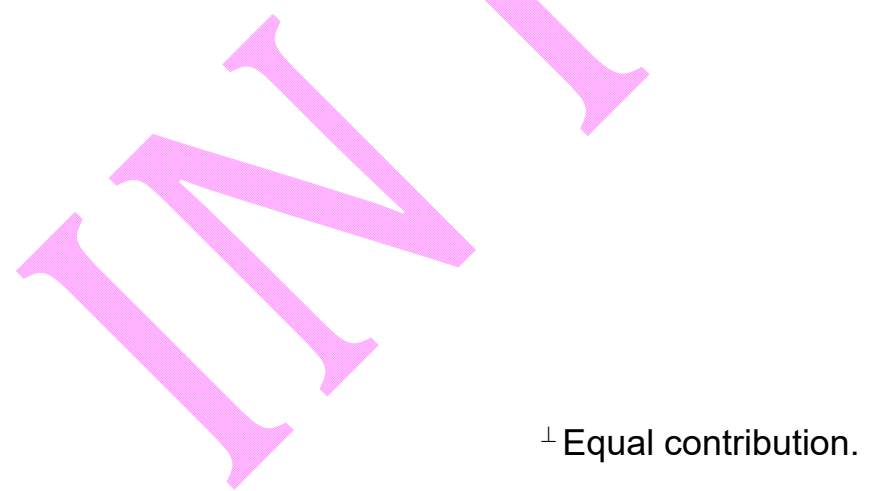 \\ * Corresponding Authors: \\ i.melian-cabrera@aston.ac.uk; ignacio.melian@gmail.com (Ignacio V. Melian Cabrera)
}




\section{Abstract}

An alternative clean regeneration method to calcination on special grade resins, i.e. Nafion, is introduced. The reactivation strategy makes use of advanced oxidation processes (Fenton and non-Fenton) to remove the organic deposits generated from sequential catalytic cycles. Hot water treatment was considered as a control case to evaluate the extraction capacity of water itself at the oxidative conditions. Advanced oxidation processes were effective in reactivating the Nafion SAC-13 resin, which also rendered a cleaner and more sustainable reactivation process. Beta zeolite was studied as model fouled system prior to the Nafion SAC-13. Even though zeolites are considered to be thermally stable, this approach can be used when a full preservation of the acid sites is required. As far as resin Nafion SAC-13 is concerned, organic species deposition was found to be responsible of a selective poisoning of the sulfonic groups in Nafion, with a consequent drop in catalytic activity on the octanoic acid esterification with methanol. The Nafion resin was reactivated either with $\mathrm{H}_{2} \mathrm{O}_{2}$ or with Fenton chemistry; the resin remained stable under these oxidative conditions, which is the benefit of the presented non-thermal methodologies as compared to calcination. The optimal method showed full recovery of the initial activity and $90 \%$ of the final conversion. This methodology seems attractive for a whole-range of organic catalytic reactions, including those related with biomass valorization, that require the use of highly acidic catalysts, such as acidic resins, in liquid phase reactions.

\section{Highlights}

- Alternative mild regeneration method to calcination for superacid resin-catalysts.

- Fenton and non-Fenton oxidation remove organics after reaction.

- Superb reactivation efficiency on the esterification of octanoic acid with methanol.

- Cleaner process than hot-water extraction in terms of residual liquids' TOC. 


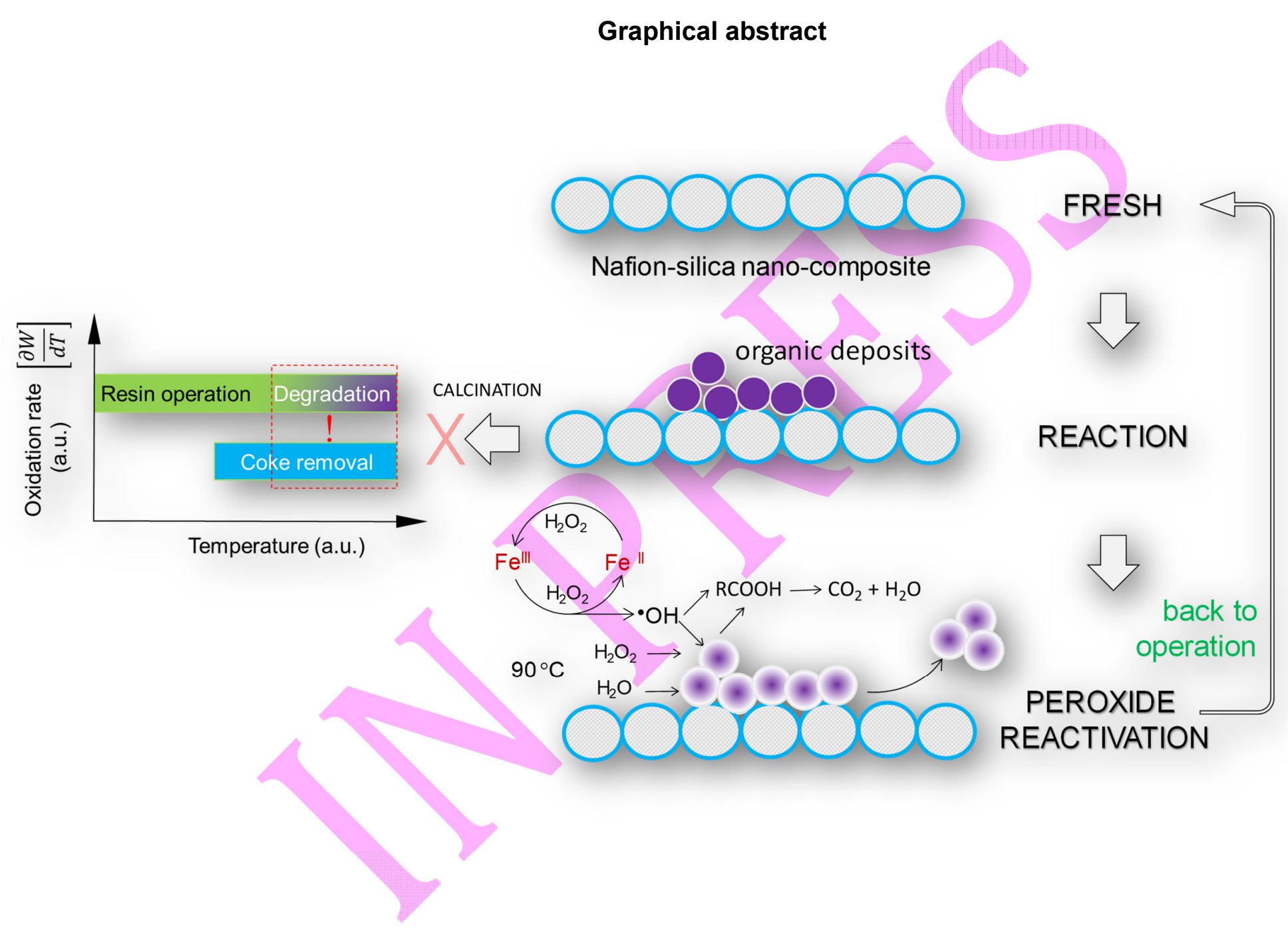

